\title{
NUMERICAL SOLUTION AND ANALYSIS FOR ACUTE AND CHRONIC HEPATITIS B
}

\section{MUHAMMAD FARMAN, ZAFAR IQBAL, AQEEL AHMAD*, ALI RAZA AND EHSAN UL HAQ}

\begin{abstract}
Department of Mathematics and Statistics, The University of Lahore, Lahore, Pakistan.
\end{abstract}
*Corresponding author: aqeelahmad.740@gmmail.com

\begin{abstract}
In this article, we present the transmission dynamic of the acute and chronic hepatitis B epidemic problem to control the spread of hepatitis B in a community. In order to do this, first we present sensitivity analysis of the basic reproduction number $R_{0}$. We develop a unconditionally convergent nonstandard finite difference scheme by applying Mickens approach $\phi(h)=h+O\left(h^{2}\right)$ instead of $h$ to control the spread of this infection, treatment and vaccination to minimize the number of acute infected, chronically infected with hepatitis B individuals and maximize the number of susceptible and recovered individuals. The stability analysis of the scheme has been developed by theorems which shows the both stable locally and globally. Comparison is also made with standard nonstandard finite difference scheme. Finally numerical simulations are also established to investigate the influence of the system parameter on the spread of the disease.
\end{abstract}

\section{INTRODUCTION}

The scope of mathematics includes mathematical modeling and esoteric mathematics. The flow of work, process, predictions and outcomes can easily be measured with the help of mathematical concepts and theory. Therefore, biologists are now extremely dependent on mathematics. Mathematical modeling of biological sciences is done by many brilliant scientist [1-3]. The relationship between simple mathematical modeling involves biological system, integer order differential equations that show their dynamics and complex system

Received 2018-06-26; accepted 2018-09-09; published 2018-11-02.

2010 Mathematics Subject Classification. 37C75, 65L07.

Key words and phrases. sensitivity analysis; acute hepatitis B; chronic hepatitis B; NSFD.

(C) 2018 Authors retain the copyrights of their papers, and all open access articles are distributed under the terms of the Creative Commons Attribution License. 
which describes their changing of structure. The nonlinearity and multi-scale behaviors in mathematical modeling describe the mutual relationship between parameter [4]. In last few decades, many biological models were studied in detail by using classical derivative, few of them in $[5,6]$.

Hepatitis B is a potentially life-threatening liver infection caused by the hepatitis B virus. It is a major global health problem. It can cause chronic liver disease and chronic infection and puts people at high risk of death from cirrhosis of the liver and liver cancer [7]. Infections of hepatitis B occur only if the virus is able to enter the blood stream and reach the liver. Once in the liver, the virus reproduces and releases large numbers of new viruses into the blood stream [8].

This infection has two possible phases: (1) acute and (2) chronic. Acute hepatitis B infection lasts less than six months. If the disease is acute, your immune system is usually able to clear the virus from your body, and you should recover completely within a few months. Most people who acquire hepatitis B as adults have an acute infection. Chronic hepatitis B infection lasts six months or longer. Most infants infected with HBV at birth and many children infected between 1 and 6 years of age become chronically infected [7]. About two-thirds of people with chronic HBV infection are chronic carriers. These people do not develop symptoms, even though they harbor the virus and can transmit it to other people. The remaining one-third develop active hepatitis, a disease of the liver that can be very serious. More than 240 million people have chronic liver infections. About 600000 people die every year due to the acute or chronic consequences of hepatitis B $[7,9]$

HBV can be transmitted from one individual to another individual on different ways, such as transmission of blood, semen and vaginal secretions [20,21,22]. Another major transmission of HBV is the unprotected sexual contact, sharing of razors, blades or tooth brushes [3].Also the virus transmits from an infected mother to her child during the time of birth. However, HBV cannot be transmitted through water, food, hugging, kissing and causal contact such as in the work place, school, etc. [22]. The mode of transmission of HBV and HIV is the same, but HBV is 50100 times more infectious [25]. HBV infection is a global health problem. According to WHO about 400million population is infected world wide chronically. In China 93million population are affected due to HBV infections $[23,27,28]$. Vaccine for the prevention of hepatitis B is available in the market that is very effective $[24,26]$. In the real world phenomena mathematical modeling is one of the powerful tools to describe the dynamical behavior of different diseases $[16,17,18,19,29]$.

Mathematical models have been used to help understand the dynamics of viral infections, such as human immunodeficiency virus and hepatitis $\mathrm{C}$ infection $[11,12]$. Following these approaches, dynamic models were developed to analyze the changes in hepatitis B virus levels during drug therapy $[13,14,15,10]$. In this article,we develop a HBV transmission model. The infectious class is divided into two stages, such as acute infectious and chronic infectious stage. Thus, the total population is divided into four compartments, $S(t)$ 
susceptible, $I_{1}(t)$ infected with acute hepatitis B, $I_{2}(t)$ infected with chronic hepatitis B and $R(t)$ recovered individuals.

In this paper, we investigate the stability and qualitative analysis of acute and chronic hepatitis B model. An unconditionally convergent nonstandard finite difference scheme has been presented to obtain solution of model. The analysis of two different states disease free and endemic equilibrium which means the disease dies out or persist in a population has been made by finding reproductive number. Numerical results are presented graphically to show the dynamics of the model.

\section{Materials And Method}

we used a mathematical model for HBV transmission by extending the work presented in [28]. We divide the host population denoted by $\mathrm{T}(\mathrm{t})$ into four compartments: susceptible individuals $S(t)$, who are not infective but have the chance to catch the disease; infected $I_{1}(t)$ represents those individuals who are infective with acute hepatitis; $I_{2}(t)$ are those individuals, who are infected with chronic hepatitis and $R(t)$ represents those individuals who have recovered after the infection with a life-time immunity. The flowchart for the transmission of HBV is given in Figure 1.

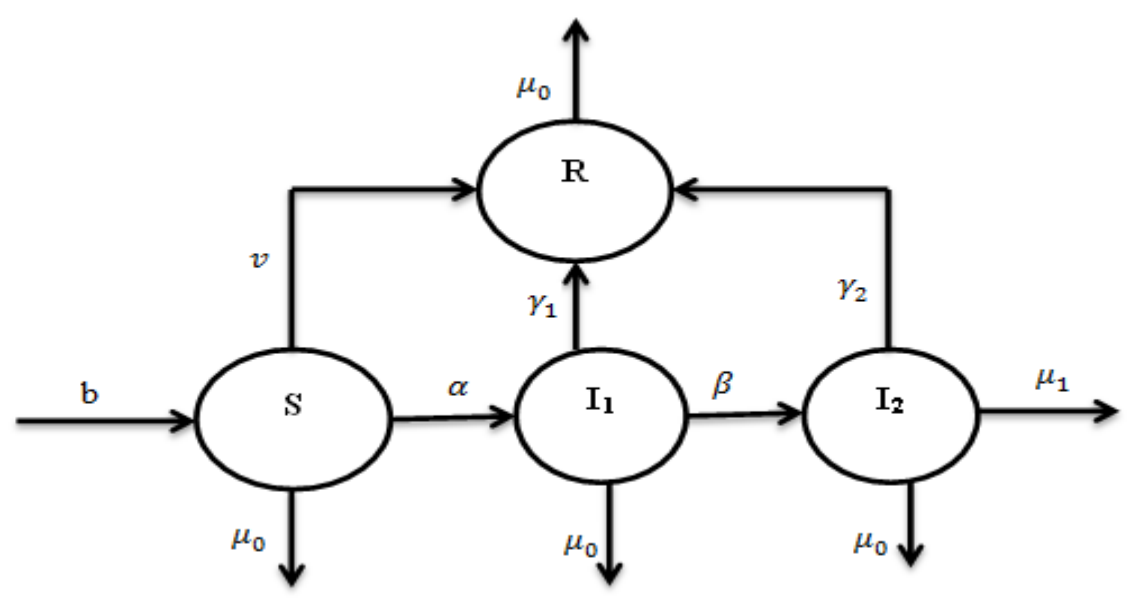

Figure 1. The flowchart of the model

Thus, the mathematical model is represented by the following four differentials equations:

$$
\begin{gathered}
\frac{d S}{d t}=b-\alpha S(t) I_{2}(t)-\left(\mu_{0}+\nu\right) S(t) \\
\frac{d I_{1}}{d t}=\alpha S(t) I_{2}(t)-\left(\mu_{1}+\beta+\gamma_{1}\right) I_{1}(t)
\end{gathered}
$$




$$
\begin{gathered}
\frac{d I_{2}}{d t}=\beta I_{1}(t)-\left(\mu_{0}+\mu_{1}+\gamma_{2}\right) I_{2}(t) \\
\frac{d R}{d t}=\gamma_{1} I_{1}(t)+\gamma_{2} I_{2}(t)+\nu S(t)-\mu_{0} R(t)
\end{gathered}
$$

with initial conditions $S(0) \geq 0, I_{1}(0) \geq 0, I_{2}(0) \geq, R(0) \geq 0$, Here $b$ represents the birth rate, $\alpha$ is the moving rate from susceptible to infected with acute hepatitis $B, \beta$ is the moving rate from acute stage to infected with chronic hepatitis, $\gamma_{1}$ is the recovery rate from acute stage to recovered, $\gamma_{2}$ is the recovery rate from chronic stage to recovered compartment, $\mu_{0}$ is the death rate occurring naturally, which is also called natural mortality rate, $\mu_{1}$ is the death rate occurring due to hepatitis $\mathrm{B}$ and $\nu$ represents hepatitis $\mathrm{B}$ vaccination rate.

\section{Qualitative Analysis}

The model $(2.1-2.4)$ is locally asymptotically as well as globally asymptotically stable at disease-free and endemic equilibrium points [29]. For disease-free equilibrium the model $(2.1-2.4)$ is both locally and globally stable, if the value of basic reproduction number is less than unity while for the endemic equilibrium the model is stable if the value of the basic reproduction number $R_{0}$ is greater than unity. Model has a disease-free equilibrium, denoted by $E_{0}$ and defined as, $E_{0}=\left(S_{0}, 0,0, R^{0}\right)$, where

$$
S_{0}=\frac{b}{\mu_{0}+\nu}
$$

and

$$
R^{0}=\frac{\nu b}{\mu_{0}\left(\mu_{0}+\nu\right)} .
$$

The endemic equilibrium is given by $E^{*}=\left(S^{*}, I_{1}^{*}, I_{2}^{*}, R^{*}\right)$, where

$$
\begin{gathered}
S^{*}=\frac{1}{\alpha \beta}\left(\mu_{0}+\beta+\gamma_{1}\right)\left(\mu_{0}+\mu_{1}+\gamma_{2}\right) \\
I_{1}^{*}=\frac{1}{\alpha \beta}\left(\mu_{0}+\nu\right)\left(\mu_{0}+\mu_{1}+\gamma_{2}\right)\left[R_{0}-1\right] \\
I_{2}^{*}=\frac{1}{\alpha}\left(\mu_{0}+\nu\right)\left[R_{0}-1\right] \\
R^{*}=\frac{1}{\mu_{0}}\left[\left(\frac{\gamma_{1}}{\alpha \beta}\left(\mu_{0}+\nu\right)\left(\mu_{0}+\mu_{1}+\gamma_{2}\right)+\frac{\gamma_{2}}{\alpha}\left(\mu_{0}+\nu\right)\left[R_{0}-1\right]\right)+\frac{\nu}{\alpha \beta}\left(\mu_{0}+\beta+\gamma_{1}\right)\left(\mu_{0}+\mu_{1}+\gamma_{2}\right)\right]
\end{gathered}
$$

Regarding these equilibrium point of the model $(2.1-2.4)$, we have the following results which are proved in $[29]$. 
3.1. Reproductive Number. Basic reproduction number $R_{0}$ is defined to be the expected number of secondary infections produced by an index case or the average number of secondary infection arising from a single individual introduced into the susceptible class during its entire infectious period in a totally susceptible population. The basic reproduction number $R_{0}$ of the model $(2.1-2.4)$ in [29] is

$$
R_{0}=\frac{\alpha \beta b}{\left(\mu_{0}+\nu\right)\left(\mu_{0}+\beta+\gamma_{1}\right)\left(\mu_{0}+\mu_{1}+\gamma_{2}\right)}
$$

Theorem 3.1. If $R_{0}<1$, then the model (2.1-2.4) is locally asymptotically stable at disease-free equilibrium, $E_{0}=\left(\frac{b}{\mu_{0}+\nu}, 0,0, \frac{\nu b}{\mu_{0}\left(\mu_{0}+\nu\right)}\right)$, while $E_{0}$ is unstable saddle point if $R_{0}>1$.

Theorem 3.2. If $R_{0} \leq 1$, then the model $(2.1-2.4)$ is globally asymptotically stable at disease-free equilibrium, $E_{0}=\left(S_{0}, 0,0, R_{0}\right)$ and unstable otherwise.

Theorem 3.3. The endemic equilibrium state $E_{1}=\left(S^{*}, I_{1}^{*}, I_{2}^{*}, R^{*}\right)$ of the model $(2.1-2.4)$ is globally asymptotically stable, if $R_{0}>1$, otherwise unstable.

Prof of these theorems will be given in [29], used in section 4.

3.2. Sensitivity Analysis of $R_{0}$ : The sensitivity of

$$
R_{0}=\frac{\alpha \beta b}{\left(\mu_{0}+\nu\right)\left(\mu_{0}+\beta+\gamma_{1}\right)\left(\mu_{0}+\mu_{1}+\gamma_{2}\right)}
$$

to each of its parameters is

$$
\begin{aligned}
& \frac{\partial R_{0}}{\partial \alpha}=\frac{\beta b}{\left(\mu_{0}+\nu\right)\left(\mu_{0}+\beta+\gamma_{1}\right)\left(\mu_{0}+\mu_{1}+\gamma_{2}\right)} \geq 0 \\
& \frac{\partial R_{0}}{\partial \beta}=\frac{\alpha b\left(\mu_{0}+\gamma_{1}\right)}{\left(\mu_{0}+\nu\right)\left(\mu_{0}+\beta+\gamma_{1}\right)^{2}\left(\mu_{0}+\mu_{1}+\gamma_{2}\right)} \geq 0 \\
& \frac{\partial R_{0}}{\partial b}=\frac{\alpha \beta}{\left(\mu_{0}+\nu\right)\left(\mu_{0}+\beta+\gamma_{1}\right)\left(\mu_{0}+\mu_{1}+\gamma_{2}\right)} \geq 0 \\
& \frac{\partial R_{0}}{\partial \nu}=-\frac{\alpha \beta b}{\left(\mu_{0}+\nu\right)^{2}\left(\mu_{0}+\beta+\gamma_{1}\right)\left(\mu_{0}+\mu_{1}+\gamma_{2}\right)} \leq 0 \\
& \frac{\partial R_{0}}{\partial \gamma_{1}}=-\frac{\alpha \beta b}{\left(\mu_{0}+\nu\right)\left(\mu_{0}+\beta+\gamma_{1}\right)^{2}\left(\mu_{0}+\mu_{1}+\gamma_{2}\right)} \leq 0 \\
& \frac{\partial R_{0}}{\partial \gamma_{2}}=-\frac{\alpha \beta b}{\left(\mu_{0}+\nu\right)\left(\mu_{0}+\beta+\gamma_{1}\right)\left(\mu_{0}+\mu_{1}+\gamma_{2}\right)^{2}} \leq 0 \\
& \frac{\partial R_{0}}{\partial \mu_{1}}=-\frac{\alpha \beta b}{\left(\mu_{0}+\nu\right)\left(\mu_{0}+\beta+\gamma_{1}\right)\left(\mu_{0}+\mu_{1}+\gamma_{2}\right)^{2}} \leq 0 \\
& \frac{\partial R_{0}}{\partial \mu_{0}}=\frac{-\alpha \beta b\left[\left(\mu_{0}+\nu\right)\left(\mu_{0}+\beta+\gamma_{1}\right)+\left(\mu_{0}+\nu\right)\left(\mu_{0}+\mu_{1}+\gamma_{2}\right)+\left(\mu_{0}+\beta+\gamma_{1}\right)\left(\mu_{0}+\mu_{1}+\gamma_{2}\right)\right]}{\left(\mu_{0}+\nu\right)^{2}\left(\mu_{0}+\beta+\gamma_{1}\right)^{2}\left(\mu_{0}+\mu_{1}+\gamma_{2}\right)^{2}} \leq 0
\end{aligned}
$$

It can be seen that $R_{0}$ is most sensitive to change in parameter, here, $R_{0}$ is increasing with $\alpha, b, \beta$, and decreasing with $\gamma_{1}, \gamma_{2}, \nu, \mu_{0}, \mu_{1}$. In other words it found that the sensitivity analysis shows that prevention is better than to control the disease. 


\section{Nonstandard Finite Difference (NSFD) Scheme}

A nonstandard finite difference (NSFD) scheme for the system $(2.1-2.4)$ is presented in this section [30]. In recent years, nonstandard finite difference (NSFD) scheme for discrete models have been constructed or tested for a wide range of nonlinear systems of differential equations $[31,32,33]$. The positivity of the state variables involved in the system is satisfy by proposed method. This property has key role when we solve mathematical models arising in biology because these state variables represent sub-populations which never take negative values. The discretized form of the the system $(2.1-2.4)$ by using NSFD scheme which based on the generalized first order forward method is written as

$$
\begin{gathered}
\frac{S^{k+1}-S^{k}}{h}=b-\alpha s^{k+1} I_{2}^{k}-\left(\mu_{0}+\nu\right) S^{k+1} \\
S^{k+1}+h \alpha s^{k+1} I_{2}^{k}+h\left(\mu_{0}+\nu\right) S^{k+1}=S^{k}+b h \\
S^{k+1}=\frac{S^{k}+b h}{1+h \alpha I_{2}^{k}+h\left(\mu_{0}+\nu\right)} \\
\frac{I_{1}^{k+1}-I_{1}^{k}}{h}=\alpha I_{2}^{k} S^{k+1}-\left(\beta+\mu_{0}+\gamma_{1}\right) I_{1}^{k+1} \\
I_{1}^{k+1}+h\left(\beta+\mu_{0}+\gamma_{1}\right) I_{1}^{k+1}=I_{1}^{k}+h \alpha I_{2}^{k} S^{k+1} \\
I_{1}^{k+1}=\frac{I_{1}^{k}+h \alpha I_{2}^{k} S^{k+1}}{1+h\left(\beta+\mu_{0}+\gamma_{1}\right)} \\
R^{k+1}\left(1+h \mu_{0}\right)=R^{k}+h\left(\gamma_{1} I_{1}^{k+1}+\gamma_{2} I_{2}^{k+1}+\nu S^{k+1}\right) \\
\frac{R_{2}^{k+1}-R^{k}}{h}=\gamma_{1} I_{1}^{k+1}+\gamma_{2} I_{2}^{k+1}+\nu S^{k+1}-\mu_{0} R^{k+1} \\
h
\end{gathered}
$$


4.1. Proposed NSFD Scheme. In this section, we design an NSFD scheme [34] that replicates the dynamics of the continuous model $(2.1-2.4)$. Let $Y_{k}=\left(S_{k}, I_{1 k}, I_{2 k}, R_{k}\right)^{T}$ denoted an approximation of $X\left(t_{k}\right)$ where $t_{k}=k \Delta t$, with $k \in \mathcal{N}, h=\Delta t>0$ be a step size then

$$
\begin{gathered}
\frac{S^{k+1}-S^{k}}{\phi}=b-\alpha s^{k+1} I_{2}^{k}-\left(\mu_{0}+\nu\right) S^{k+1} \\
\frac{I_{1}^{k+1}-I_{1}^{k}}{\phi}=\alpha I_{2}^{k} S^{k+1}-\left(\beta+\mu_{0}+\gamma_{1}\right) I_{1}^{k+1} \\
\frac{I_{2}^{k+1}-I_{2}^{k}}{\phi}=\beta I_{1}^{k+1}-\left(\mu_{1}+\mu_{0}+\gamma_{2}\right) I_{2}^{k+1} \\
\frac{R^{k+1}-R^{k}}{\phi}=\gamma_{1} I_{1}^{k+1}+\gamma_{2} I_{2}^{k+1}+\nu S^{k+1}-\mu_{0} R^{k+1}
\end{gathered}
$$

which is the new purposed NSFD scheme for the given model, where

$$
\phi=\phi(h)=\frac{1-e^{-\left(\beta+\mu_{0}+\gamma_{1}\right) h}}{\beta+\mu_{0}+\gamma_{1}}
$$

The discrete method $(4.13-4.16)$ is indeed an NSFD scheme because it is constructed according to Mickens rules [33] formalized as follows in [34].

Rule 1. The standard denominator $h=\Delta t$ of the discrete derivatives is replaced by the complex denominator function in Equation (4.17) which satisfies the asymptotic relation

$$
\phi(h)=h+O\left(h^{2}\right)
$$

Note that the denominator function $\phi$ is expected to better capture the dynamics of the continuous model through the presence of the underlying parameters $\mu_{0}, \beta, \gamma_{1}$. In fact, exact schemes for a wide range of dynamical systems involve such complex denominator functions $[35,36]$.

Rule 2.Nonlinear terms in the right-hand side of Equation $(2.1-2.4)$ are approximated in a non-local way. For instance, we have $I_{2}\left(t_{k}\right) S\left(t_{k}\right) \simeq I_{2 k} S_{k+1}$ instead of $I_{2}\left(t_{k}\right) S\left(t_{k}\right) \simeq I_{2 k} S_{k}$

\subsection{Analysis of the Scheme.}

Theorem 4.1. The NSFD scheme $(4.13-4.16)$ is a dynamical system on the biological feasible domain $\mathcal{K}$ of the continuous model $(2.1-2.4)$.

Proof:First, we prove the positivity of the scheme $(4.13-4.16)$. It is easy to show that the NSFD scheme $(4.13-4.16)$ takes the explicit form

$$
S^{k+1}=\frac{S^{k}+\phi b}{1+\alpha \phi I_{2}^{k}+\left(\mu_{0}+\nu\right) \phi}
$$




$$
\begin{gathered}
I_{1}^{k+1}=\frac{\left[1+\alpha \phi I_{2}^{k}+\left(\mu_{0}+\nu\right) \phi\right]\left[I_{1}^{k}+\alpha \phi\left(S^{k}+\phi b\right) I_{2}^{k}\right]}{\left[1+\phi\left(\mu_{0}+\beta+\gamma_{1}\right)\right]\left[1+\alpha \phi I_{2}^{k}+\left(\mu_{0}+\nu\right) \phi\right]} \\
I_{2}^{k+1}=\frac{\left[1+\phi\left(\mu_{0}+\beta+\gamma_{1}\right)\right]\left[1+\alpha \phi I_{2}^{k}+\left(\mu_{0}+\nu\right) \phi\right]\left(I_{2}^{k}+\beta \phi I_{1}^{k}\right)+\beta \alpha \phi^{2}\left(S^{k}+\phi b\right) I_{2}^{k}}{\left[1+\phi\left(\mu_{0}+\mu_{1}+\gamma_{2}\right)\right]\left[1+\phi\left(\mu_{0}+\beta+\gamma_{1}\right)\right]\left[1+\alpha \phi I_{2}^{k}+\left(\mu_{0}+\nu\right) \phi\right]} \\
R^{k+1}=\frac{R^{k} \cdot A \cdot B \cdot C \cdot D+\phi\left\{\gamma_{1}\left(\text { A.D.C } I_{1}^{k}+\alpha \phi \cdot E\right)+\gamma_{2} A\left(B \cdot C\left[I_{2}^{k}+\beta \phi I_{1}^{k}\right]+\alpha \beta \phi^{2} I_{2}^{k} \cdot E\right)+\nu E \cdot A \cdot B \cdot C\right\}}{A \cdot B \cdot C \cdot D}
\end{gathered}
$$

where

$$
\begin{gathered}
A=1+\mu_{0} \phi, B=1+\phi\left(\mu_{0}+\beta+\gamma_{1}\right), C=1+\alpha \phi I_{2}^{k}+\left(\mu_{0}+\nu\right) \phi \\
D=1+\phi\left(\mu_{0}+\mu_{1}+\gamma_{2}\right), E=S^{k}+\phi b
\end{gathered}
$$

Thus $S^{k+1} \geq 0, I_{1}^{k+1} \geq 0, I_{2}^{k+1} \geq 0, R^{k+1} \geq 0$ whenever the discrete variables are non-negative at the previous iteration. It remains to prove the positive invariance of $\mathcal{K}$. Adding the (4.13) and (4.14), we have

$$
\begin{gathered}
{\left[1+\phi\left(\mu_{0}+\nu\right)\right] H^{k+1}=\phi b+H^{k}-\left[1+\left(\mu_{0}+\mu_{1}+\gamma_{1}\right) \phi\right] I^{k} \leq \phi b+H^{k}} \\
{\left[1+\phi\left(\mu_{0}+\nu\right)\right] H^{k+1} \leq \phi b+H^{k}} \\
\Rightarrow H^{k+1} \leq \frac{b}{\mu_{0}+\nu}
\end{gathered}
$$

whenever

$$
H^{k} \leq \frac{b}{\mu_{0}+\nu}
$$

The priori bonds for $I_{2}^{k+1}$ and $R^{k+1}$ follow the radially from the fact that $I_{2}^{k+1}$ and $I_{1}^{k+1}$ and less then or equal $H^{k+1}$. This complete the proof.

Theorem 4.2. (1) The disease-free fixed point (resp. the endemic fixed point) of the NSFD scheme (4.134.16) for the model without recruitment/provision of disease is GAS whenever $R_{0} \leq 1$ (resp. whenever $\left.R_{0}>1\right)$.

(2) The endemic fixed-point of the NSFD scheme $(4.13-4.16)$ for the full model is GAS.

Proof:Let $Y_{k} \in \mathcal{R}_{+}^{4}$ be the bounded sequence defined by the NSFD scheme $(4.13-4.16)$. We want to prove that $Y_{k}$ tends to $Y^{*}$, where $Y^{*}$ is any of the fixed point. By Bolzano Weierstrass theorem, there exists a subsequence $Y_{n_{k}}$ of $Y_{n}$ that converge to some $Z^{*}$ as $k \rightarrow+\infty$. By the assumption made above and the structure of the NSFD scheme $(4.13-4.16), Y^{*}=Z^{*}$ is necessarily either the unique disease-free fixed-point 
$E_{0}$ (whenever $\left.R_{0} \leq 1\right)$ or the unique endemic fixed-point $E^{*}$ or the unique endemic $E^{\diamond}$, which is LAS thanks to Theorem 4.2. Therefore, there exists $\theta>0$ such that for an initial condition $Y_{0}$ satisfying

$$
\left\|Y^{0}-Y^{*}\right\| \leq \theta
$$

we have

$$
\lim _{x \rightarrow+\infty}\left\|Y^{0}-Y^{*}\right\|=0
$$

let $Y^{0}$ be an arbitrary initial condition. As

$$
\lim _{x \rightarrow+\infty} Y_{n_{k}}=Y^{*}
$$

there exits a integer $k_{0}$ such that

$$
\left\|Y_{n_{k 0}}-Y^{*}\right\| \leq \theta
$$

In view equation (4.18) and (4.19), we have

$$
\lim _{x \rightarrow+\infty, n \geq 1}\left\|Y_{n_{n}}-Y^{*}\right\|=\lim _{x \rightarrow+\infty, n \geq n_{k_{0}}}\left\|Y_{n_{n}}-Y^{*}\right\|=0
$$

This prove that $Y^{*}$ is GAS.

TABLE 1. Values of physical parameters used in model when $R_{0}<1$

\begin{tabular}{|c|c|c|c|}
\hline Parameter & Value & Parameter & Value \\
\hline$n_{1}$ & 100 & $n_{2}$ & 40 \\
\hline$n_{3}$ & 20 & $n_{4}$ & 5 \\
\hline$b$ & 0.4 & $\nu$ & 0.02 \\
\hline$\beta$ & 0.01 & $\mu_{0}$ & 0.03 \\
\hline$\mu_{1}$ & 0.002 & $\gamma_{1}$ & 0.05 \\
\hline$\gamma_{2}$ & 0.06 & $\alpha$ & 0.005 \\
\hline
\end{tabular}


TABLE 2. Values of physical parameters used in model when $R_{0}>1$

\begin{tabular}{|c|c|c|c|}
\hline Parameter & Value & Parameter & Value \\
\hline$n_{1}$ & 100 & $n_{2}$ & 40 \\
\hline$n_{3}$ & 20 & $n_{4}$ & 5 \\
\hline$b$ & 0.4 & $\nu$ & 0.02 \\
\hline$\beta$ & 0.1 & $\mu_{0}$ & 0.03 \\
\hline$\mu_{1}$ & 0.04 & $\gamma_{1}$ & 0.05 \\
\hline$\gamma_{2}$ & 0.06 & $\alpha$ & 0.05 \\
\hline
\end{tabular}

4.3. Numerical Simulations. The mathematical analysis of epidemic model hepatitis B with non-linear incidence has been presented. To observe the effects of the parameters using in this dynamics hepatitis B model $(2.1-2.4)$, conclude several numerical simulations varying the value of parameters given in table 1 and table 2 for $R_{0}<1$ and $R_{0}>1$ respectively. Figure 2 and 3 shows the convergence solution for diseases free and endemic equilibria by using NSFD scheme at $h=1$. Figure 4 and 5 also represent the he convergence solution for diseases free and endemic equilibria by using NSFD scheme at $\phi=\phi(h)+O\left(h^{2}\right)$. The technique create a better impact to control the hepatitis B, it reduces the infected rate and increases the susceptible and recovered population during disease free state as well as in endemic state.

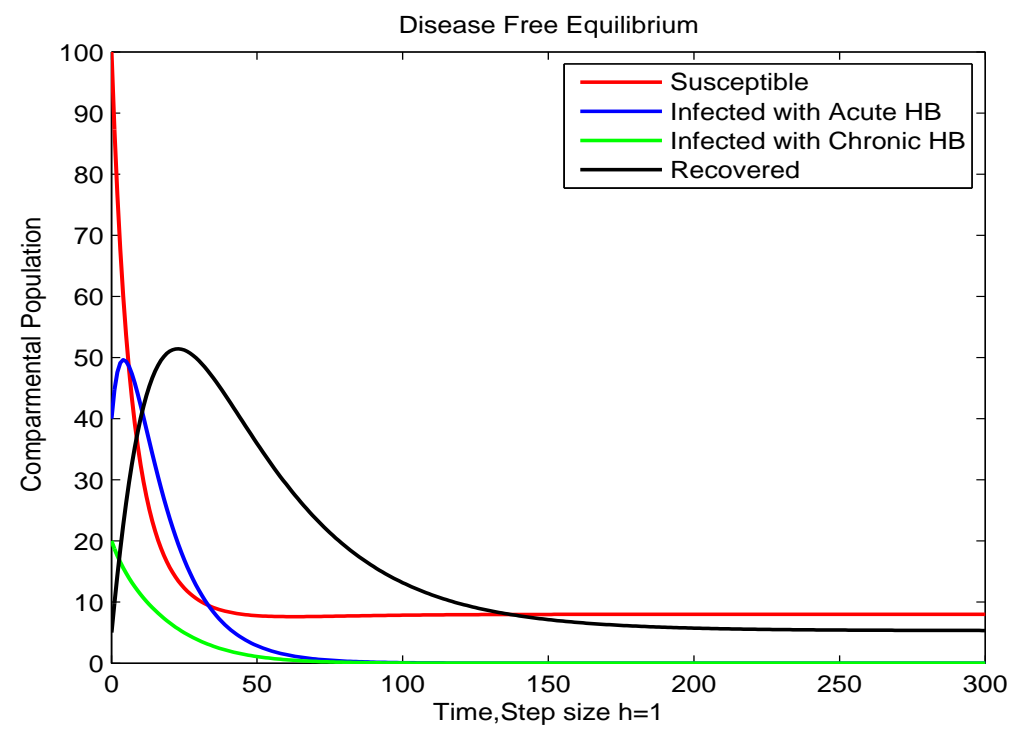

FigurE 2. Numerical solutions for susceptible, acute infected individual, chronic infected individual and recovered population in a time $t$ with step size $h=1$ for disease free equilibrium points. 


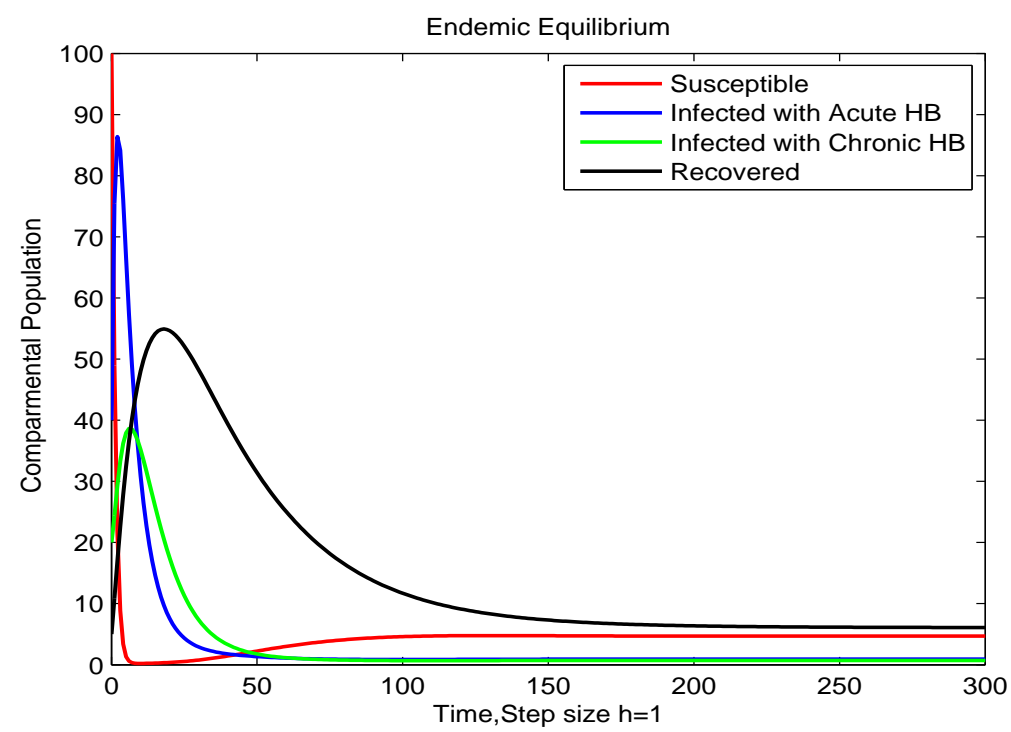

Figure 3. Numerical solutions for susceptible, acute infected individual, chronic infected individual and recovered population in a time $t$ with step size $h=1$ for endemic equilibrium points.

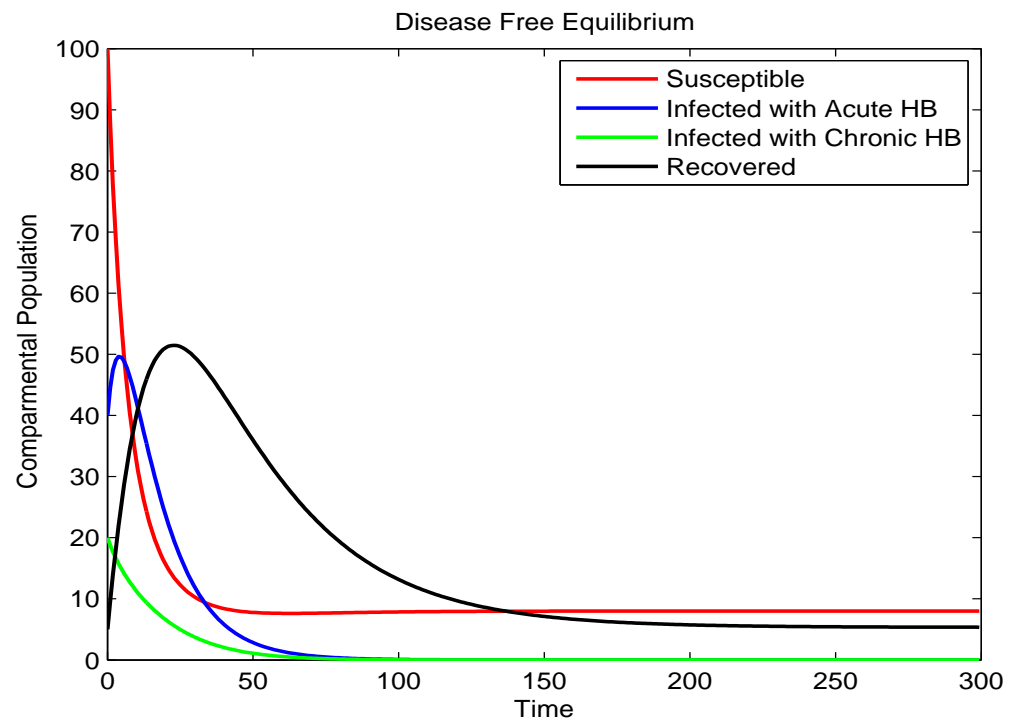

FiguRE 4. Numerical solutions for susceptible, acute infected individual, chronic infected individual and recovered population in a time $t$ by using $\phi=\phi(h)$ with step size $h=1$ for disease free equilibrium points. 


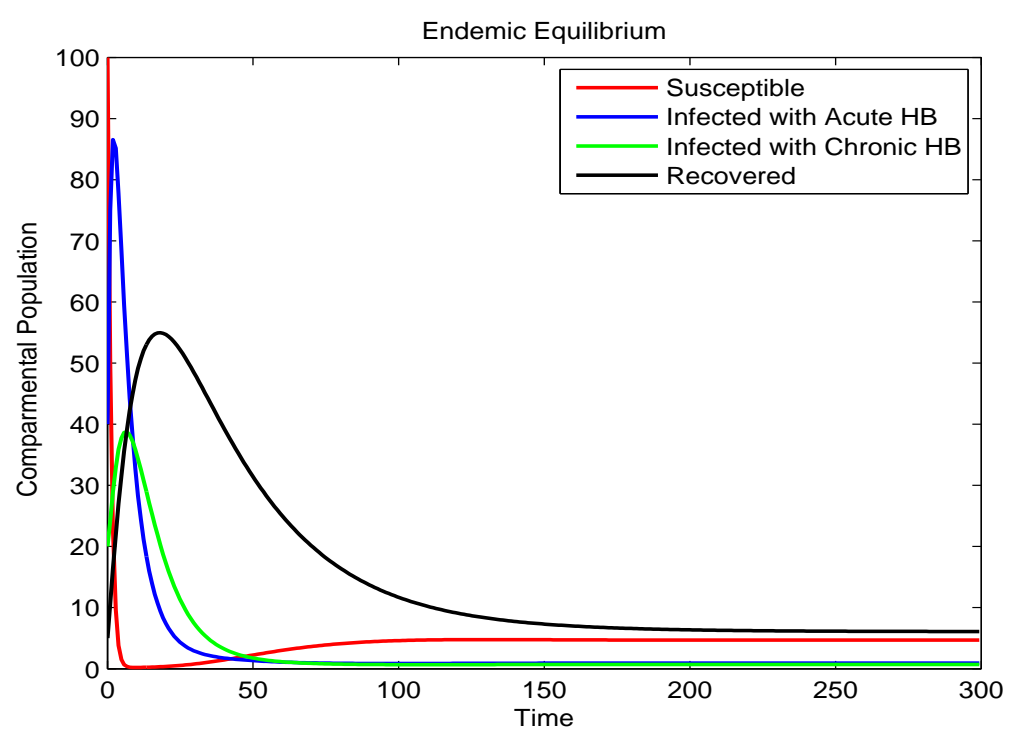

Figure 5. Numerical solutions for susceptible, acute infected individual, chronic infected individual and recovered population in a time $t$ by using $\phi=\phi(h)$ with step size $h=1$ for endemic equilibrium points.

\section{Conclusion}

We have considered a mathematical system of equation which describes the hepatitis B disease. The analysis of the system is well established. Sufficient conditions for local stability of the DFE point $E_{0}$ are given in terms of the basic reproduction number $R_{0}$ of the model, where it is asymptotically stable if $R_{0}<1$. The positive infected equilibrium $E^{*}$ exist when $R_{0}>1$ and sufficient conditions that guarantee the asymptotic stability of the point are given. Beside this sensitivity analysis of the parameters involved in threshold parameter $R_{0}$ is discussed. It is important to note that nonstandard finite difference scheme for mathematical models based on system of differential equations is more powerful approach to compute the convergent solutions for the disease models. The nonstandard finite difference scheme is dynamically consistent, easy to implement and show a good agreement to control the bad impact of hepatitis B for long period of time and to eradicate a death killer factor in the world spread by hepatitis B. Finally, we presented the numerical simulation and verified all the analytical results numerically by using nonstandard finite difference scheme to reduce acute as well chronic infected rates for both disease free and endemic equilibria, we are able to control the spreading of hepatitis B in the community.

\section{REFEREnCES}

[1] J. Biazar, Solution of the epidemic model by Adomian decomposition method, Appl. Math. Comput. 173 (2006), 1101-1106. 
[2] S. Busenberg, P. Driessche, Analysis of a disease transmission model in a population with varying size, J. Math. Biol. 28 (1990), 65-82.

[3] A.M.A. El-Sayed, S.Z. Rida and A.A.M. Arafa, On the solutions of time-fractional bacterial chemotaxis in a diffusion gradient chamber, Int. J. Nonlinear Sci. 7 (2009), 485-495.

[4] O.D. Makinde, Adomian decomposition approach to a SIR epidemic model with constant vaccination strategy, Appl. Math. Comput. 184 (2007), 842-848.

[5] A.A.M. Arafa, S.Z. Rida and M. Khalil, Fractional modeling dynamics of HIV and 4 T-cells during primary infection, Nonlinear Biomed. Phys. 6 (2012), 1-7.

[6] C.M. Kribs-Zaleta, Structured models for heterosexual disease transmission, Math. Biosci. 160 (1999), 83-108.

[7] B. Buonomo and D. Lacitignola, On the dynamics of an SEIR epidemic model with a convex incidence rate, Ricerche Mat. 57 (2008), 261-281.

[8] WHO, Hepatitis B Fact Sheet No. 204, The World Health Organisation, Geneva, Switzerland, 2013, http://www.who.int/mediacentre/factsheets/fs204/en/.

[9] Canadian Centre for Occupational Health and Safety, Hepatitis B, http : //www.ccohs.ca/oshanswers/diseases/hepatitis . html.

[10] A. V. Kamyad, R. Akbari, A. K Heydari and A. Heydari Mathematical Modeling of Transmission Dynamics and Optimal Control of Vaccination and Treatment for Hepatitis B Virus, Comput. Math. Methods Med. 2014 (2014), Article ID 475451.

[11] C.M. Stanca, R.M. Ruy, W.N. Patrick and S.P. Alan, Modeling the mechanisms of acute hepatitis B virus infection, J. Theor. Biol. 247 (2007), 23-35.

[12] A. Perelson, Modelling viral and immune system dynamics. Nature Rev. Immunol. 2 (2002), 28-36.

[13] A. Perelson and R. Ribeiro, Hepatitis B virus kinetics and mathematical modeling. Sem. Liv. Dis. 24 (2004), $11-15$.

[14] M. Nowak, S. Bonhoeffer,A. Hill, R. Boehme, H. Thomas, H. McDade, Viral dynamics in hepatitis B infection. Proc. Natl Acad. Sci. USA 93 (1996), 4398-4402.

[15] S. Lewin, R. Ribeiro, T. Walters, G. Lau, S. Bowden, S. Locarnini and A. Perelson, Analysis of hepatitis B viral load decline under potent therapy: complex decay profiles observed. Hepatology 34 (2001), 1012-1020.

[16] P. Colombatto, L. Civitano, R. Bizzarri, F. Oliveri, S. Choudhury, R. Gieschke, F. Bonino and M.R. Brunetto, A multiphase model of the dynamics of HBV infection in HBeAg-negative patients during pegylated interferon-a2a, lamivudine and combination therapy. Antiviral Therapy 11 (2006), 197-212.

[17] J. Wang, J. Pang and X. Liu,Modelling diseases with relapse and nonlinear incidence of infection: A multi-group epidemic model, J. Biol. Dyn. 8 (2014), 99-116.

[18] J. Wang, R. Zhang and T. Kuniya, The stability analysis of an SVEIR model with continuous age-structure in the exposed and infectious classes, J. Biol. Dyn. 9 (2015), 73-101.

[19] G. Zaman, Y.H. Kang and I.H. Jung, Stability and optimal vaccination of an SIR Epidemic Model, BioSystems 93 (2008), 240249 .

[20] G. Zaman, Y.H. Kang and I.H. Jung, Optimal treatment of an SIR epidemic model with time delay, BioSystems 98 (2009), 43-50.

[21] D. Lavanchy, Hepatitis B virus epidemiology, disease burden, treatment, and current and emerging prevention and control measures, J. Viral Hepat. 11 (2004), 97-107.

[22] A.S. Lok, E.J Heathcote and J.H. Hoofnagle, Management of hepatitis B, 2000 Summary of a workshop, Gastroenterology 120 (2001), 18281853.

[23] B.J.McMahon, Epidemiology and natural history of hepatitis B, Semin. Liver Dis. 25 (2005), 38. 
[24] M.K. Libbus and L.M. phillips, Public health management of perinatal hepatitis B virus, Public Health Nurs. 26 (2009), 353-361.

[25] J.E. Maynard, M.A. Kane and S.C. Hadler, Global control of hepatitis B through vaccination role of hepatitis B vaccine in the expanded programme on immunization, Rev. Infect. 2 (1989), S574-S578.

[26] S. Thornley, C. Bullen and M. Roberts,Hepatitis B in a high prevalence NewZealand population: A mathematical model applied to infection control policy, J. Theor. Biol. 254 (2008), 599-603.

[27] C.W. Shepard, E.P. Simard, L. Finelli, A.E. Fiore and B.P. Bell, Hepatitis B virus infection epidemiology and vaccination, Epidemiol. Rev. 28 (2006), 112-125.

[28] R. Williams, Global challenges in liver disease, Hepatology 44 (2006), 521-526.

[29] T. Khan, G. Zaman and M. I. Chohan, The transmission dynamic and optimal control of acute and chronic hepatitis B, J. Biol. Dyn. 11(2017), 172-189.

[30] R. E. Mickens, Exact solutions to a finite difference model of a nonlinear reactions advection equation: Implications for numerical analysis, Numer. Methods Partial Differ. Equ. 5 (1989), 313-325.

[31] R. E. Mickens, Applications of Nonstandard finite difference Schemes, World Scientific, Singapore (2000).

[32] R. Anguelov and J.M.S Lubuma, Nonstandard finite difference method by nonlocal approximations, Math. Comput. Simul. 61 (2003), 465-475.

[33] R.E. Mickens, Nonstandard finite difference Models of differential equations, World Scientific, Singapore (1994).

[34] R. Anguelov and J.M.S. Lubuma, Contributions to the mathematics of the nonstandard finite differencemethodandapplications, Numer. Methods Partial Differ. Equ. 17 (2001), 518-543.

[35] J.M.S. Lubuma and K.C. Patidar, Non-standard methods for singularly perturbed problems possessing oscillatory/layer solutions, Appl. Math. Comput. 187(2) (2007), 1147-1160.

[36] L.W. Roeger, Exact difference schemes, in A. B. Gumel Mathematics of Continuous and Discrete Dynamical Systems, Contemp. Math., Vol. 618, Amer. Math. Soc., Providence, RI, (2014), 147-161. 